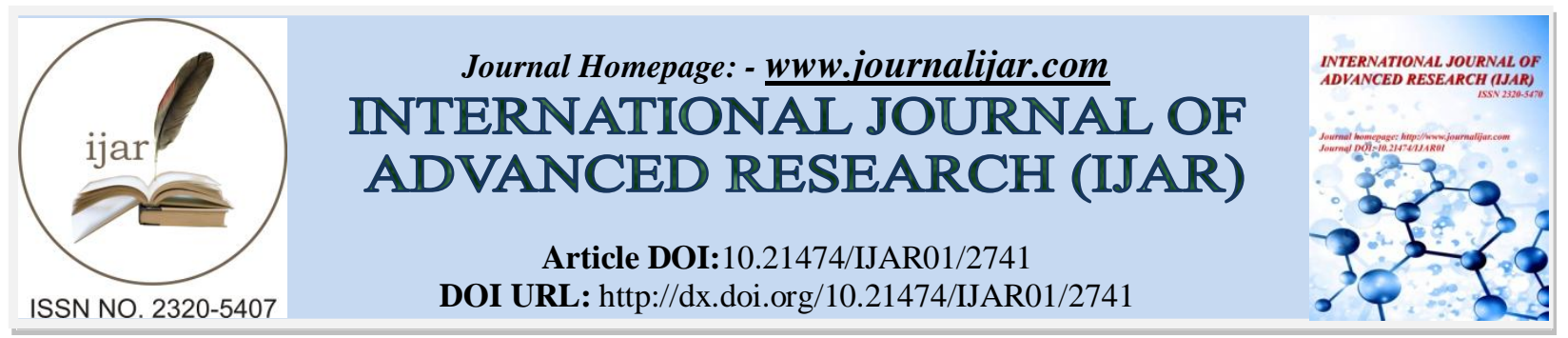

RESEARCH ARTICLE

\title{
CORONARY ARTERY DISEASE IN RHEUMATOID ARTHRITIS - PREVALENCE,PATHOPHYSIOLOGY,RISK AND TREATMENT
}

1. Pri-Med Care, Lewisville Texas, USA

\author{
Maria Aziz ${ }^{1 *}$ and K.S. Yadav².
}

2. RGCP, Bhopal, India *Corresponding author: Maria Aziz, Pri-Med Care, Lewisville Texas, USA

\section{Manuscript Info}

..........................

Manuscript History

Received: 15 November 2016

Final Accepted: 17 December 2016

Published: January 2017

\section{Abstract}

Coronary Artery Disease (CAD), is a significant contributor morbidity and mortality in Rheumatoid Arthritis ( RA) . ${ }^{1}$ Familiarity with the pathophysiology of coronary atherosclerosis will have important practical implications for patient care .Insights in to the mechanisms of thrombosis will give further insight into the concomitant therapy used during acute revascularization. ${ }^{2}$ The purpose of this review is thorough understanding of the connection between atherosclerosis and RA and stress in accurate risk stratification in RA patients and timely intervention to improve outcomes. The review also highlights comprehensive collaboration between Rheumatologist and cardiologist to help in prevention of cardiovascular diseases in RA patients and improve patient care in a total spectrum.

Copy Right, IJAR, 2016,. All rights reserved.

\section{Introduction:-}

Rheumatoid Arthritis ( RA),is the most common autoimmune articular disease with systemic manifestations.It affects 2,1 million Americans and 1.5 million women .It is more common in females. The reduced life span of RA patients is possibly explained by the cardiovascular diseases. ${ }^{3}$

Coronary Artery Disease (CAD), is a significant contributor morbidity and mortality in RA ${ }^{4}$ Rheumatoid arthritis patients have increased risk of Myocardial Infarction. Hence aggressive interventional prevention strategies are implicated in RA patients with cardiovascular diseases.

There is adeep connection between CAD and RA .Thorough understanding of this connection will help in accurate risk stratification in RA patients and timely intervention will improve outcomes ,Comprehensive collaboration between Rheumatologist and cardiologist will help in prevention of cardiovascular diseases in RA patients and will improve patient care in a total spectrum.

Atherosclerosis has inflammatory origin. ${ }^{5}$ Similarity has been observed between pathology mechanism underlying in the synovium of joints of RA patients and endothelium of arteries in atherosclerosis.In both atherosclerosis and RA, there is infiltration of inflammatory cells, dysfunctioning of endothelial cells and synovial cells,smooth cell proliferation .The hallmark mechanism underlying both diseases is Inflammation. ${ }^{6}$

Chief contributors of atherosclerosis in RA are-Endothelial dysfunction caused by immune complexes,antibodymediated,cytokinemediated,Autoantibodies,hypercoagulability,dyslipidemia.Other traditional 
risk factors of consideration are hypertension , diabetes, raised body mass index ,hyperhomocystinemia.:There is also association of the HLA-DRB1 gene with premature death, particularly from cardiovascular disease, in patients with rheumatoid arthritis. ${ }^{7}$

Previously,our understanding of atherosclerosis was different as it is today .Earlier it was considered a cholesterol storage disease, but now our understanding about its pathophysiology has remarkably evolved and now its considered an inflammatory disease .CAD causes stenosis hence need of revascularization to relieve ischemia..Besides treatment of stenosis through revascularization ,aggressive management of modifiable risk factors is also important .Disrupted plaques are the stimulus for thrombosis .Its a solid state stimulus .Changes in levels of circulating prothrombotic or antifibrinolytic mediators in blood predispose to acute coronary syndrome .Widespread inflammation and multiple high risk plaques predispose to ACS ,ACS is no more a localized or segmental disease. The goal of treatment should be treating early lesions and stabilizing other plaques to prevent recurrent events in the future .Treatment of stenosis should include mechanical revascularization and further preventive interventions to prevent future events. ${ }^{8-10}$

\section{Discussion:-}

\section{Pathophysiology of chronic cad:-}

Atherogenesis is a complex interaction of risk factors ,cells of the arterial wall and inflammatory mediators and proinflammatory cytokines .Inflammation plays role in all stages of atherosclerosis1

Inflammation also plays role in blood vessel ,cardiac and systemic complications of atherosclerosis .

The chief key players involved in the pathomechanisms are circulating cytokines,tissue necrosis factor alpha.,INFgamma,interleukin 1 , interleukin 6,hyperhomocysteinemia,ESR,CRP .

Endothelial dysfunction first marks the first step of atherosclerosis. ${ }^{11}$ It is the earliest event that signals the beginning of atherosclerosis.Exposure of arterial endothelium to proinflammatory cytokines derived from excess adipose tissue ,products of glycoxidation associated with hyperglycemia, vasoconstrictor hormones in hypertension,dyslipidemia and bacterial products, leads to expression of adhesion molecules.${ }^{12}$ These adhesion molecules allow blood leukocytes to stick to the inner surface of the arterial wall .Next step is the transmigration of the adherent leukocytes. The leukocytes reside in the intima and communicate with the endothelial cells and smooth muscle cells . ${ }^{12}$ Mediators of inflammation that are involved in this process are prostanoids, leukotrienes ,histamine cytokines,protein mediators and complement components ${ }^{14,16}$ As a next sequel ,SMC s migrate from the tunica media to the intima. These secrete extracellular matrix .,SMC, endothelial cells and monocytes secrete MMP in response to hemodynamic stresses .MMP $\mathrm{s}$ play vital role in regulating functions of vascular cells , angiogenesis, healing, destruction of extracellular matrix of arteries.Proteoglycans of extracellular matrix binds to lipoproteins, prolong their residence in the intima ,render them susceptible to glycation and oxidative modifications .Lipoprotein modification gives products such as oxidized phospholipids and advanced glycation end products .They propagate inflammatory response .Next step of progression is the calcification and the bone formation ${ }^{15}$ There is both proliferation and death of lipid laden macrophages. The extracellular lipid coalesce in the intima and form lipid rich necrotic core of atherosclerotic plaque .

The atherosclerotic lesion grows outward rather than inward .Thus stenosis is the last stage of atherosclerosis .By the time stenosis is diagnosed and treated, atherosclerosis already had a widespread and diffuse distribution .Thus the prevalence of atherosclerosis is still underscored and underdiagnosed specially in adolescent and young adult Americans . ${ }^{17}$

Complications in plaques like disruption of plaque, superficial erosion ,intraplaque hemorrhage and erosion of calcified nodule account for all acute coronary thromboses.Disrupted plaques lead to thrombosis . When the plaque disrupts, collagen is exposed and this exposed collagen in the extracellular matrix triggers platelet activation .Smooth muscle cells and macrophages secrete TF and it activates the coagulation cascade. Thus disrupted plaque is a stimulus to both thrombosis and coagulation .Thrombin amplifies platelet activation .Fibrinogen is converted to fibrin .Activated platelets release vWF.vWF helps in the formation of white arterial thrombus which is a dense mesh of fibrin network in which platelets are entrapped.This is the solid state of the disrupted plaque .In addition to this we have the fluid phase also which predisposes to coronary thrombosis .Raised levels of plasminogen activator 
inhibitor -1 ,specially in diabetes, obesity and hypertensive patients is responsible for fluid phase changes .Disrupted plaque plaque secrete particulate $\mathrm{TF}$ which increase thrombogenicity of blood.$^{18-19}$

Plaques with thin fibrous cap and a large lipid core with numerous inflammatory cells and few SMCs are prone for rupture.These are high risk plaques.Patients with high risk plaques and wide spread inflammation are prone to acute coronary syndrome .Plaques with outward remodeling lead to hidden lesions that lack angiographic detection but harbor a substantial burden of atherosclerosis. They donotcause symptoms nor do they cause ischemia.Myeloperoxidase is a marker of inflammation and is raised in ischemia.Diffuse arterial inflammation is responsible for focal lesions and clinical presentations

\section{Signs and symptoms:-}

The key clinical manifestations of CAD are stable angina pectoris , unstable angina pectoris ,MI.Chest discomfort is the first symptomatic episode. ${ }^{20}$ Sometimes the first coronary artey diagnostic presentation is acute plaque rupture or acute myocardial infarction .Cardiac dysrhythmias represent underlying electrical instability.Angina pectoris is a cardinal symptom developed due to inadequate oxygen supply to the myocardium.Cardinal angina symptoms are discomfort in arm , throat, jaw,exertional fatigue ,shortness of breath,heartburn. Angina Pectoris is said to be stable when its pattern of duration, intensity, frequency are not changed over time .Administration of sublingual nitroglycerin can help to differentiate stable and accelerating pattern.

Accelerating Angina shows a change in pattern of stable Angina.Easyprovocation,more prolonged episodes,more intensity ,longer recovery and more often use of nitroglycerin.Its a transitioning stage from stable to unstable angina.Transition from Stable Angina to accelerating pattern warrants medical attention.

Unstable Angina ,pattern of chest discomfort changes abruptly. Chest pain at rest, increase in frequency, discomfort with minimal activity. Unstable Angina occurs when there is sudden rupture of atherosclerotic plaqueor abnormal occlusion of coronary artery .MI follows as asequale of unstable angina.Immediate medical attention is warranted.

Clinical symptoms pertaining to metabolic syndrome should also be evaluated and be a part of work up plan ${ }^{21}$

\section{Diagnosis:-}

Key diagnostic approach is detail patient history,through physical examination,EKG,laboratory blood tests ,stress test and cardiac catheterization. Imaging methods help to assess the regional myocardial perfusion and function. New imaging technologies that provide risk of progression and further cardiovascular events are optical coherence tomography,thermography,Infrared spectroscopy ,electron beam computed tomography,MRI. ${ }^{22}$

\section{Treatment:-}

The aim of treatment is to reduce myocardial oxygen requirements and increase the myocardial blood flow .Medications that reduce oxygen requirements are nitroglycerin ,nitrates ,Beta blockers and calcium channel blockers .Revascularization procedures thrive on the concept of restoring forward coronary artery blood flow .New procedures of revascularization include arteriogenesis stimulation by cells,protein and gene.Besides treatment of flow limiting lesions ,non obstructive plaques should also be addressed.Angiography helps to assess the severity of lesions .Ischemia is the end result of dynamic obstruction superimposed on fixed stenosis .Sudden rapid progression of the lesion leads to poor prognosis .Fixed stenosis progress in sudden spurts ${ }^{23}$ There is discontinuous progression of plaques .Sudden increase in the severity of the obstruction is due to episodes plaque disruption ,thrombosis and healing .Revascularization procedures relieve ischemia but donot protect against future acute thrombotic events .Modification of risk factors delay disease progression. Thus for atherosclerotic patients combination of treatment strategies are used ,revascularization procedures, lifestyle modifications and pharmacological measures .Modification of risk factors include -reduction in blood pressure, reduction in LDL , smoking cessation .Diabetes Mellitus ,Metabolic syndrome lead to adverse lipid profile and dyslipidemia leading to CVD events. ${ }^{24}$

Primary prevention of cardiovascular disease is lifestyle modification. The hear protection study promotes the use of statins in individuals with total cholesterol $>135 \mathrm{mg} / \mathrm{dl}$ and with traditional risk factors . The physician's health study ,showed aspirin significantly reduces MI in men aged 40-80 yrs 20 .EUROPA study showed that ACE inhibitor significantly reduce CVS events .Besides pharmacological therapies, lifestyle modification carry equal importance .Biomarkers linked to inflammation have been studied and it was found that they predict coronary events better than traditional risk factors.24These markers include acute phase reactants,cytokines ,MMPs.Patients with elevated CRP 
at baseline are benefited most by aspirin and statin therapy in primary prevention trials .They reduce CRP and other markers of inflammation .This supports the beneficial use of anti-inflammatory in atherosclerosis. ${ }^{25}$

Bypass surgery and percutaneous revascularization treat localized segmental disease .The goal of treatment should be to restrict thrombosis and embolism both ,maintain perfusion and limit loss of cardiomyocytes..Severe ischemia leads to ST-T segment abnormalities and release of troponin T or I . These are signs of poor prognosis .Aggressive management should include platelet inhibition, inhibition of thrombin generation revascularization of culprit lesions .This approach will improve outcome in high risk patients.Best combination recommended for high risk patients is oral aspirin ,clopidogrel and intravenous glycoproteinIIb/IIIa during angioplasty during angioplasty ${ }^{26}$

Through understanding of pathophysiology of ACS will help to reduce plaque atherogenicity , achieve rapid control of disease process,prevent future recurrences.Use of statins helps to prevent recurrences of ACS in the future by reducing cholesterol levels and by anti-inflammatory actions.COX-2 inhibitors also retard atherosclerosis

Management of ACS should aim to stabilize lesions .Stabilization of lesions reduce recurrent events .Systemic factors that cause plaque disruption should be adequately controlled ..Statins by affecting the biology of the plaque reduce recurrent coronary events.55.Inflammation underlies the mechanism of plaque formation hence anti inflammatory will have beneficial effects 56.Statins reduce CRP levels ,hence improve outcomes in ACS .

Prime aim of treatment should be to stabilize lesions and control all systemic factors adequately that leads thrombotic complications of atherosclerosis .Both size of the plaque and composition of the plaque decides future complications .Larger plaques cause stenosis and ischemia .Smaller plaques could grow oblong towards the outer wall and can be silent killers and cannot be detected on the angiogram. Sturdier extracellular matrix skeleton are less likely to rupture and less likely to trigger clot formation .

\section{Conclusions:-}

Our understanding about CAD has significantly changes in the last few decades .Now atherosclerosis is not merely just a cholesterol storage disease but its more of an inflammatory disease .Familiarity with the pathophysiology of coronary atherosclerosis will have important practical implications for patient care .Insights in to the mechanisms of thrombosis will give further insight into the concomitant therapy used during acute revascularization .Therapies should be individualized based on patient's specific characteristics..Preventive therapy should be emphasized in more efficient and cost effective manner.LDL should be targetized to reduce risk of atherosclerotic complications.Lifestyle modifications with drug treatments should be used patients with obesity,metabolic syndrome and diabetes.

\section{References:-}

1. Solomon DH, Karlson EW, Rimm EB, et al: Cardiovascular morbidity and mortality in women diagnosed with rheumatoid arthritis. Circulation 2003;107:1303-1307.

2. Edwards CJ, Syddall H, Goswami R, et al: The autoantibody rheumatoid factor may be an independent risk factor for ischaemic heart disease in men. Heart 2007;93(10):1263-1267.

3. Heliövaara M, Aho K, Knekt P, et al: Rheumatoid factor, chronic arthritis and mortality. Ann Rheum Dis 1995;54(10):811-814.

4. Wallberg-Jonsson S, Ohman ML, Dahlqvist SR: Cardiovascular morbidity and mortality in patients with seropositive rheumatoid arthritis in Northern Sweden. J Rheumatol 1997;24:445-451.

5. Libby P: Inflammation in atherosclerosis. Nature 2002;420(6917):868-874.

6. Jawien J: New insights into immunological aspects of atherosclerosis. Pol Arch Med Wewn 2008;118(3):127131.

7. Farragher TM, Goodson NJ, Naseem H, et al: Association of the HLA-DRB1 gene with premature death, particularly from cardiovascular disease, in patients with rheumatoid arthritis and inflammatory polyarthritis. Arthritis Rheum 2008;58(2):359-369.

8. Harvey EJ, Ramji DP: Interferon-gamma and atherosclerosis: Pro- or anti-atherogenic? Cardiovasc Res 2005;67(1):11-20

9. Shah PK: Role of inflammation and metalloproteinases in plaque disruption and thrombosis. Vasc Med 1998;3(3):199-206. 
10. Aubry MC, Riehle DL, Edwards WD, et al: B-Lymphocytes in plaque and adventitia of coronary arteries in two patients with rheumatoid arthritis and coronary atherosclerosis: Preliminary observations. CardiovascPathol 2004;13(4):233-236.

11. Pober JS: Effects of tumour necrosis factor and related cytokines on vascular endothelial cells. Ciba Found Symp 1987;131:170-184.

12. Pasceri V, Willerson JT, Yeh ET: Direct proinflammatory effect of C-reactive protein on human endothelial cells. Circulation 2000; 102(18):2165-2168.

13. Aziz M, Yadav KS. Atherosclerosis: An extra articular Manifestation of Rheumatoid Arthritis AnnClin Lab Res. 2016, 4: 4.

14. Aziz M, Yadav KS (2016) Role of Traditional Risk Factors, Inflammation and DMARDs in Cardiovascular Diseases in Rheumatoid Arthritis and Management of Lipid profile J Autoimmune Disord 2: 3. doi: 10.21767/2471-8513.100026

15. Aziz M, Yadav KS. Pathogenesis of Atherosclerosis. Med Clin Rev. 2016, 2:3.

16. Chung CP, Oeser A, Solus JF, et al: Inflammation-associated insulin resistance: Differential effects in rheumatoid arthritis and systemic lupus erythematosus define potential mechanisms. Arthritis Rheum 2008;58(7):2105-2112.

17. Yalamanchili K, Aronow WS, Kilaru R, et al: Coronary artery disease is more severe in older persons with rheumatoid arthritis than in older persons without rheumatoid arthritis. Cardiol Rev 2006;14(2):55-56.

18. Turesson C, McClelland RL, Christianson TJ, Matteson EL: Severe extra-articular disease manifestations are associated with an increased risk of first ever cardiovascular events in patients with rheumatoid arthritis. Ann Rheum Dis 2007;66(1):70-75.

19. Aubry MC, Maradit-Kremers H, Reinalda MS, et al: Differences in atherosclerotic coronary heart disease between subjects with and without rheumatoid arthritis. J Rheumatol 2007;34(5):937-942.

20. Asanuma Y, Chung CP, Oeser A, et al: Serum osteoprotegerin is increased and independently associated with coronary-artery atherosclerosis in patients with rheumatoid arthritis. Atherosclerosis 2007; 195(2):e135-e141.

21. Chung CP, Oeser A, Solus JF, et al: Prevalence of the metabolic syndrome is increased in rheumatoid arthritis and is associated with coronary atherosclerosis. Atherosclerosis 2008;196(2):756-763.

22. Omland T, Ueland T, Jansson AM, et al: Circulating osteoprotegerin levels and long-term prognosis in patients with acute coronary syndromes. J Am CollCardiol 2008;51(6):627-633.

23. Kao AH, Krishnaswami S, Cunningham A, et al: Subclinical coronary artery calcification and relationship to disease duration in women with rheumatoid arthritis. J Rheumatol 2008;35(1):61-69.

24. Dessein PH, Norton GR, Woodiwiss AJ, et al: Influence of nonclassical cardiovascular risk factors on the accuracy of predicting subclinical atherosclerosis in rheumatoid arthritis. J Rheumatol 2007;34(5):943-951.

25. Avalos I, Chung CP, Oeser A, et al: Increased augmentation index in rheumatoid arthritis and its relationship to coronary artery atherosclerosis. J Rheumatol 2007;34(12):2388-2394.

26. Ciftci O, Yilmaz S, Topcu S, et al: Impaired coronary microvascular function and increased intima-media thickness in rheumatoid arthritis. Atherosclerosis 2008;198(2):332-337. 